\title{
Analysis of 42 Cases of First Trimester Screening in a Private Set-up
}

\author{
${ }^{1}$ Manasi Venkatraman, ${ }^{2}$ Sameer Dikshit, ${ }^{3}$ Ketan Gundavda \\ ${ }^{1}$ Resident, BSES MG Global Hospital, Mumbai, Maharashtra, India \\ ${ }^{2}$ Consultant, Department of Fetal Medicine, BSES MG Global Hospital, Mumbai, Maharashtra, India \\ ${ }^{3}$ Chief Consultant, BSES MG Global Hospital, Mumbai, Maharashtra, India
}

Correspondence: Sameer Dikshit, Consultant, Department of Fetal Medicine, EMP 50/704, Thakur Village, Kandivali East Mumbai-400101, Maharashtra, India, e-mail: drsameerd@gmail.com

\section{ABSTRACT}

Combined NT and first trimester biochemistry for aneuploidy has become a standard practice. This study was conducted in a busy private clinic. The results of this study showed that this study can be successfully performed in a busy private clinic. The results of the combined screening match those of the conventional screening tests. Proper counseling help the women to take a decision towards invasive testing in screen positive cases.

Keywords: First trimester screening, NT, Screen positive, Invasive testing

\section{INTRODUCTION AND AIM}

Combined NT and first trimester biochemistry for aneuploidy have become the standard practice world over. This study was conducted in a busy private set-up to find out the following:

1. If this can be done in a busy private set-up

2. If the results match the standards achieved by other studies

3. What percent of screen-negative patients using only NT screening turn screen-positive, if both NT and biochemistry are used

4. What is the response of the screen-positive patients?

\section{MATERIALS AND METHODS}

This study was conducted in a single fetal medicine unit between 15 March 2010 and 31 May 2010. The patients were scanned by a sonographer who has been certified for first trimester screening by the Fetal Medicine Foundation (FMF). The patients were first scanned for viability of the pregnancy, and the crownrump length (CRL) was measured when the fetus was in neutral sagittal position. The nuchal translucency (NT) was measured when the CRL was between 45 and $84 \mathrm{~mm} .{ }^{1}$ The NT was measured following all the FMF guidelines. ${ }^{1}$ The results were entered in the FMF software for risk assessment. The baseline risk generated before entering NT measurement was labeled as background risk or baseline risk. The risk generated using only NT measurements was labeled as "NT risk". For this study, only NT measurement was considered, and nasal bone, ductus

Date of Received: 14-12-10

Date of Acceptance: 22-06-11

Date of Publication: May 2011 venosus and tricuspid regurgitation were not considered. The patient's blood was collected for free beta-hCG and PAPP-A. The blood was tested in FMF accredited laboratory (Perkin Elmer Life Sciences and Mediscan, Chennai). The biochemistry results and NT measurements were used to generate "combined risk”. Risk of one in 250 was taken as screen-positive for both NT risk and combined risk. ${ }^{3}$ The patients were counseled (by KG, MV) based on the results. The counseling included informing the patients that this was not a diagnostic test. They were also informed that there was a fair chance (depending on the risk involved) of having a chromosomally normal fetus. The screen-positive patients were offered the choice of invasive testing. The pregnancies that had high NT were also advised that even if they were screen-negative, they had a high risk of having a fetus with other genetic syndrome, a fetus with cardiac anomaly or a fetus with skeletal dysplasia. They were informed that they would need a malformation scan at 20 weeks to rule out these conditions. If they declined it, then the reasons thereof were recorded.

\section{RESULTS}

Total 42 patients were examined (Table 1). Out of these, NT could be measured in 37 patients (88\%). It could not be measured in five patients (12\%). The reasons for not measuring NT were either unfavorable fetal position or NT image not fulfilling the FMF guidelines.

The patients ranged from gestational age of 11.2 to 14.1 weeks (Table 2).

The CRL ranged from 45 to $83 \mathrm{~mm}$, and the NT ranged from 1.1 to $3.0 \mathrm{~mm}$.

Background screening was done based on maternal age, gestational age and ethnicity. Out of the total 42 cases, 37 cases 
tested screen-negative with background screening, giving a screen-negative percent of $88 \%$. Those who were screenpositive had the risks $1: 160,1: 240,1: 230,1: 160$ and 1:200 (Table 3 and Fig. 1).

Out of the 37 patients in whom NT could be measured, NT risk was calculated. There were 36 screen-negative patients (97.3\%) and one patient was screen-positive (2.7\%) (Table 4). The risk in the screen-positive patient was 1:16 (Fig. 2).

Out of the 37 patients in whom NT was done, the biochemistry was used to give a combined risk (Table 5); 33 patients were screen-negative (89\%) while four were screenpositive (11\%). The risks in screen-positive patients were 1:110, 1:55, 1:70 and 1:200 (Fig. 3).

Out of the 36 screen-negative patients after NT screening, three patients were found to be screen-positive after combined screening (Table 6 and Fig. 4).

Out of the four patients who were screen-positive, three (75\%) chose to undergo invasive testing while one (25\%) chose to terminate the pregnancy (Table 7 and Fig. 4).

Table 1: Summary of all cases

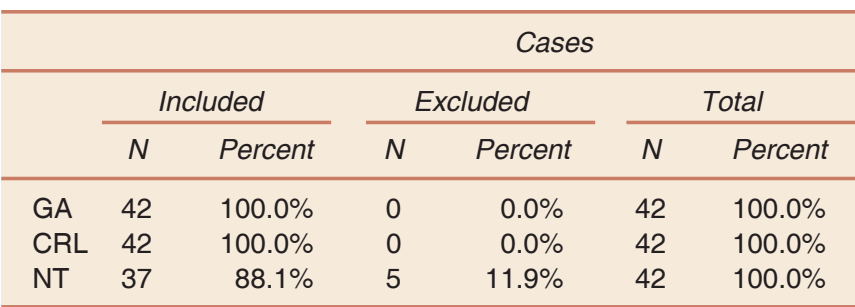

Table 2: Summary of GA, CRL and NT

\begin{tabular}{lrrr}
\hline & $G A$ & $C R L$ & $N T$ \\
\hline $\mathrm{N}$ & 42.0 & 42 & 37.0 \\
Minimum & 11.2 & 45 & 1.1 \\
Maximum & 14.1 & 83 & 3.0 \\
\hline
\end{tabular}

Summary of GA,CRL and NT

Table 3: Background screen

\begin{tabular}{llrrrr}
\hline & Frequency Percent & \multicolumn{2}{c}{$\begin{array}{c}\text { Valid } \\
\text { percent }\end{array}$} & $\begin{array}{c}\text { Cumulative } \\
\text { percent }\end{array}$ \\
& & & 88.1 & 88.1 & 88.1 \\
Valid Screen negative & 37 & 11.9 & 11.9 & 100.0 \\
Screen positive & 5 & 100.0 & 100.0 & \\
Total & 42 & 100 & \\
\hline
\end{tabular}

Table 4: NT screen

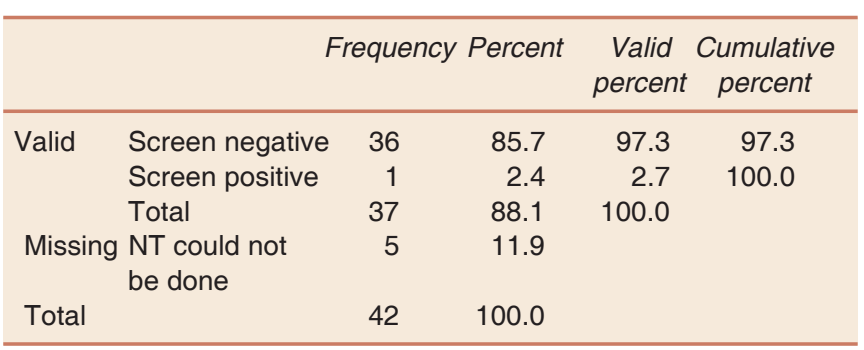

\section{DISCUSSION}

First trimester screening has become a standard practice in most of the advanced countries. NT, other sonological markers and serum biochemistry are used to give a combined risk. ${ }^{1}$ It is yet to become a standard practice in India. Alternative practice is to refer all patients more than 35 years of age for screening test. However, this is not correct practice, as in recent times, the incidence of Down syndrome babies in pregnancies in women younger than 35 years has increased to $43 \%{ }^{2}$

This study was conducted in a busy private set-up and was meant to see if the first trimester screening, as suggested by the

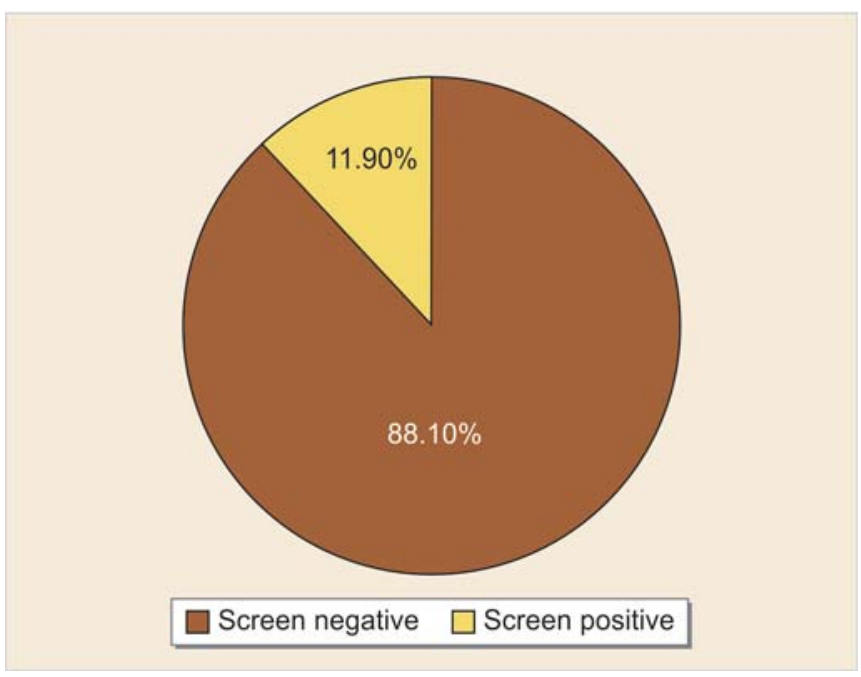

Fig. 1: Background screen

Table 5: Combined NT and biochemical screen

\begin{tabular}{lrrrrr}
\hline & Frequency & Percent & $\begin{array}{c}\text { Valid } \\
\text { percent }\end{array}$ & $\begin{array}{c}\text { Cumulative } \\
\text { percent }\end{array}$ \\
\hline Valid & Screen negative & 33 & 78.6 & 89.2 & 89.2 \\
& Screen positive & 4 & 9.5 & 10.8 & 100.0 \\
& Total & 37 & 88.1 & 100.0 & \\
Missing NT missing & 5 & 11.9 & & \\
Total & 42 & 100.0 & & \\
\hline
\end{tabular}

Table 6: Comparison of NT and combined screen

\begin{tabular}{llcc}
\hline & \multicolumn{2}{c}{ Valid combined screen } \\
\cline { 3 - 4 } & & $\begin{array}{c}\text { Screen negative } \\
\text { count }\end{array}$ & $\begin{array}{c}\text { Screen positive } \\
\text { count }\end{array}$ \\
\hline \multirow{2}{*}{ NT screen } & Screen negative & 33 & 3 \\
& Screen positive & 0 & 1
\end{tabular}

Table 7: Outcome of screen positive

\begin{tabular}{llcc}
\hline & \multicolumn{2}{c}{ Valid combined screen } \\
\cline { 3 - 4 } & & $\begin{array}{c}\text { Screen negative } \\
\text { count }\end{array}$ & $\begin{array}{c}\text { Screen positive } \\
\text { count }\end{array}$ \\
\hline \multirow{2}{*}{ Outcome } & No action & 33 & 0 \\
& Invasive testing & 0 & 3 \\
& Terminated & 0 & 1 \\
\hline
\end{tabular}




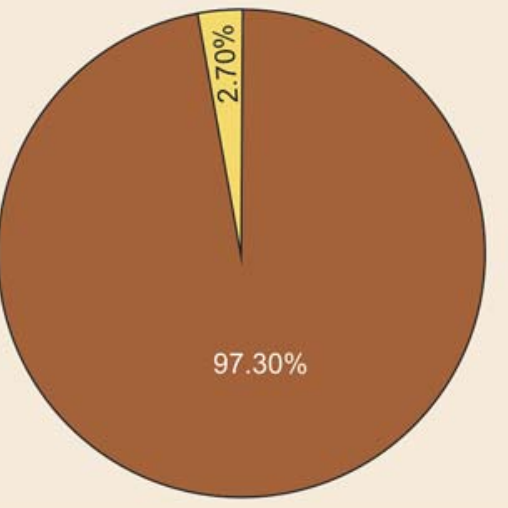

Valid screen negative $\quad \square$ Valid screen positive

Fig. 2: NT screen

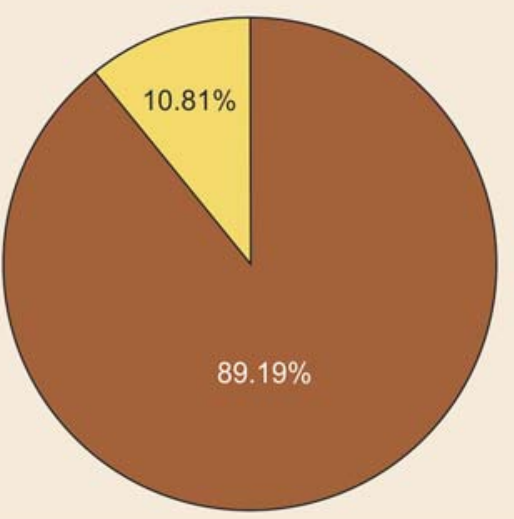

Valid screen negative $\quad \square$ Valid screen positive

Fig. 3: Valid combined screen

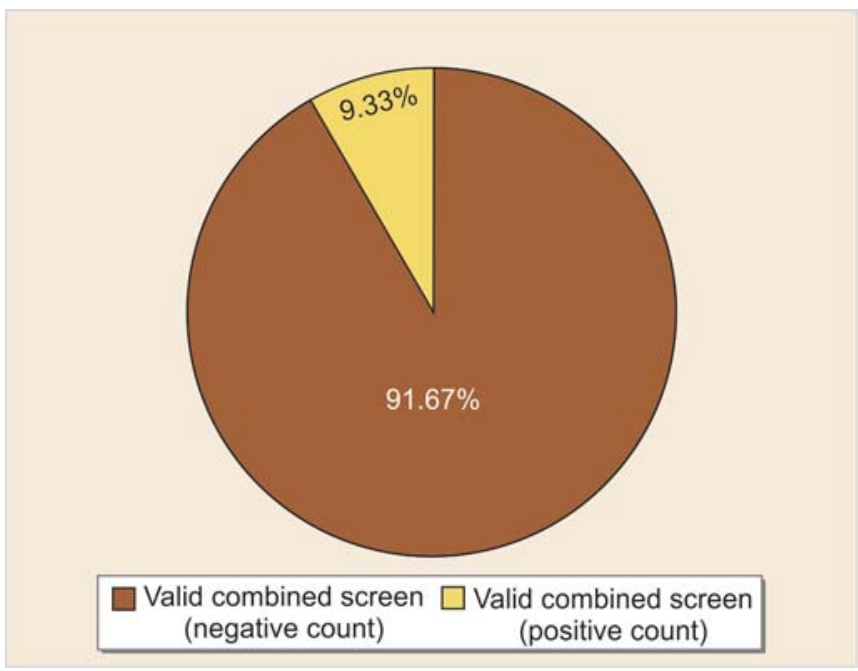

Fig. 4: Percentage of NT screen negative who turned screen positive after combined screen

FMF, could be included as a part of the routine work-up. Out of the total 42 patients scanned, NT could be measured successfully in five patients (12\%). In various studies, NT could

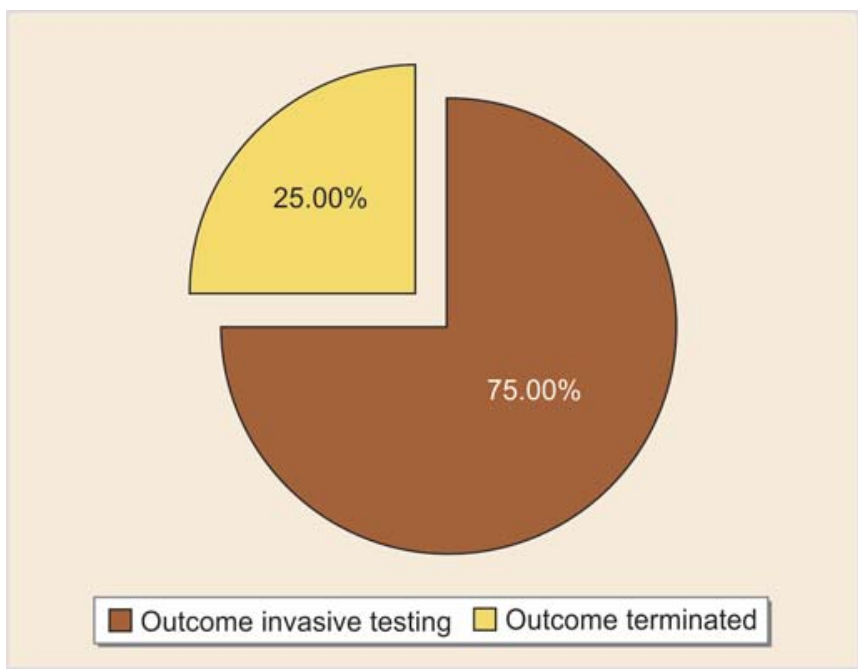

Fig. 5: Outcome of screen positive

not be measured in $20 \%$ of cases. ${ }^{3}$ In this study, the reasons why NT could not be scanned in five patients were:

a. Unfavorable fetal position and

b. FMF criteria were not satisfied in the NT image.

In this study, the background risk (a priori risk) was measured in all 42 patients; 37 patients (88\%) were screennegative and five patients (12\%) were screen-positive. Using NT measurement to modify the background risk in 37 cases where valid NT measurement could be made, one patient was deemed screen-positive (3\%) and 36 patients (97\%) were screen-negative. When serum biochemistry was added to calculate the risk in these 37 patients, 33 patients were screennegative (90\%) and four patients were screen-positive (10\%). Significantly, out of 36 screen-negative patients using NT screening, three patients who were termed screen-negative, were termed screen-positive (8\%). Various studies have proven that combined screening is superior to plain NT screening. ${ }^{4}$ There were no false-positive findings in the study but there exists potential for false-positive, if only NT screening is used in place of combined screening.

Out of four screen-positive patients after combined screening, three underwent invasive testing and one terminated the pregnancy. Those who underwent invasive testing (amniocentesis) were found to have normal karyotype (falsepositive). The reason for termination of pregnancy was social pressure. This indicated that most of the patients chose to undergo further testing instead of termination of pregnancy. ${ }^{17,18}$ The one patient who terminated the pregnancy was from poor socioeconomic class where the cost of invasive testing may have played a role in the final decision, however, the patient declined to assign any reason.

\section{SUMMARY}

1. The combined screening can be done in a busy practice, $88 \%$ of the patients could undergo NT measurement successfully.

2. At $10 \%$ screen-positive rates of the combined screening, the results match the standards achieved by other studies. 
3. $8 \%$ of screen-negative patients using only NT screening turned screen-positive, if both NT and biochemistry were used. Using combined screening is better than plain NT screening.

4. Only $25 \%$ of screen-positive patients chose termination of pregnancy. This indicates that with efficient counseling, patients accept invasive testing over termination of pregnancy.

\section{REFERENCES}

1. Spencer K, Spencer CE, Power M, Dawson C, Nicolaides KH. Screening for chromosomal abnormalities in the first trimester using ultrasound and maternal serum biochemistry in a one stop clinic: A review of 3 years prospective experience. BJOG 2003b;110:281-86.

2. Morris JK, Alberman E. Trends in Down's syndrome live births and antenatal diagnoses in England and Wales, from 1989 to 2008: Analysis of data from National Down's syndrome cytogenic register. BMJ Oct 2009;26:339.

3. Drysdale K, Ridley D, Walker K, Higgins B, Dean T. First trimester pregnancy scanning as a screening tool for high risk and abnormal pregnancies in a district general hospital setting. J Obstet Gynaecol Mar 2002;22(2):159-65.

4. Spencer K, Souter V, Tul N, Snijders R, Nicolaides KH. A screening program for trisomy 21 at 10 to 14 weeks using fetal nuchal translucency, maternal serum free $\beta$-human chorionic gonadotropin and pregnancy-associated plasma protein-A. Ultrasound in Obstetrics and Gynecology 1999;13:231-37. 